\title{
Gluon Shadowing and Nuclear Entanglement
}

\author{
Paolo Castorina ${ }^{1,2,3}$, Alfredo Iorio $^{2}$, Daniele Lanteri ${ }^{1,2,4}$ and Petr Lukeš ${ }^{2}$ \\ ${ }^{1}$ INFN, Sezione di Catania, I-95123 Catania, Italy \\ ${ }^{2}$ Faculty of Mathematics and Physics, Charles University \\ V Holešovičkách 2, 18000 Prague 8, Czech Republic \\ 3 School of Nuclear Science and Technology, Lanzhou University, \\ 222 South Tianshui Road, Lanzhou 730000, China \\ ${ }^{4}$ Dipartimento di Fisica e Astronomia, Università di Catania, I-95123 Catania, Italy.
}

(Dated: March 3, 2020)

\begin{abstract}
Relying on previous results that link entanglement entropy and parton distribution functions in deep inelastic scattering and focusing on the small Bjorken scaling region we present here indications that gluon shadowing might indeed be explained as due to a depletion of the entanglement entropy between observed and unobserved degrees of freedom per nucleon within a nucleus with respect to the free nucleon case. We apply to gluon shadowing the general Page approach to the calculation of the entanglement entropy in bipartite systems, giving physical motivations of the results.
\end{abstract}

PACS numbers: 13.60.Hb,12.38.Cy,03.65.Ud

\section{Introduction}

Quantum-Chromodynamics (QCD) evolution equations of parton distribution functions (pdfs) in deep inelastic scattering (DIS) require specific initial conditions, i.e. the evaluation of the pdfs as a function of the Bjorken scaling variable, $x$, at a fixed transfer momentum, $-q_{0}^{2}=Q_{0}^{2}$.

Ideed, the phenomenological analyses of pdfs [1, 2] are crucial for a reliable comparison of QCD predictions with the experimental data in high energy collisions, taking into account the effective uncertainty in the evolution equations.

Moreover, a quantitative description of the modifications of pdfs in nuclear environment is important to disentangle the so-called cold nuclear effects from the genuine signatures of the formation of a quark-gluon plasma (QGP) at finite temperature and baryon density in relativistic heavy ion collisions. Indeed, the well known phenomenon of "nuclear shadowing" [3, i.e. the depletion of pdfs per nucleon within a nucleus with respect to the free nucleon case in the small $x$ region can mimic the suppression of particle production due to the formation of the QGP.
The functional form of the initial pdfs is a nonperturbative problem related to quark and gluon confinement and a recent proposal [4] suggests that the gluon distribution function in the low $x$ region can be directly related to the entanglement entropy of the parton in the nucleon wave function. More precisely, the nucleon in its rest frame is described by a pure quantum mechanical state $|\psi\rangle$ with density matrix $\rho=|\psi><\psi|$, such that the entanglement entropy

$$
S=-\operatorname{Tr}(\rho \ln \rho),
$$

is identically zero. However, DIS probes only part of the nucleon's wave function, call it the spatial region $I$.

The inclusive DIS measurement thus sums over the unobserved part of the wave function localized in the region $I I$, complementary to $I$, so one has access only to the reduced density matrix, $\rho_{I}=\operatorname{Tr}_{I I} \rho$.

In [4] it turns out that in the small $x$ region $(x \leq 0.008)$ the entanglement entropy $S_{I} \equiv-\operatorname{Tr}\left(\rho_{I} \ln \rho_{I}\right)$ is related to the gluon distribution $x G(x)$ as follows

$$
\begin{aligned}
S_{I} & \equiv S_{N}(x)=\ln [x G(x)] \\
& =\Delta \ln [1 / x]=\Delta \ln [L / \epsilon],
\end{aligned}
$$


where $L=1 /\left(m_{N} x\right)$, with $m_{N}$ the nucleon's mass, $\epsilon=1 / m_{N}$ is the Compton wavelength of the nucleon and $\Delta=0.15$, for the free nucleon [5] at $Q_{0}^{2} \simeq 1.7 \mathrm{Gev}^{2}$.

Taking this point of view, gluon shadowing should be due to a reduction of the entanglement between observed and unobserved degrees of freedom (d.o.f.) for a bounded nucleon with respect to a free nucleon.

At a first glance, this conclusion could appear to contradict the idea that the entanglement entropy, $S_{I}$, for a subsystem $I$ of fixed (quantum) dimension $m$ is bigger when the combined system $I \bigcup I I$ has a bigger (quantum) dimension $N=m n$.

However, the crucial aspect is which d.o.f. are effectively entangled in any given specific process. It is the intent of this paper to propose a model-independent analysis of gluon shadowing based on entanglement entropy where a dynamical mechanism selects the effective entangled d.o.f. and produces a smaller $S_{I}$.

In Section I we recall the general argument by Page [6, 7] on the average entropy of a quantum subsystem, based on the unitary evolution of a pure state into a pure state. Section III is devoted to the application of Page's formulas of entanglement entropy to gluon shadowing. In Sec. III we discuss some physical motivations of the obtained results and Sec. IV] is devoted to comments and conclusions.

\section{ENTANGLEMENT ENTROPY AND PAGE CURVES}

Consider the Hilbert space $\mathrm{H}$ of a generic quantum bipartite system, $\mathrm{H}=\mathrm{H}_{I}^{m} \otimes \mathrm{H}_{I I}^{n}$, where $m$ and $n$ indicate the dimension, so that $\operatorname{dim} \mathrm{H}=$ $m n \equiv N$. Pick up there an arbitrary state $\mid \psi_{0}>\in \mathbf{H}$, and a random unitary matrix $U$, so that $U \mid \psi_{0}>$ is a random state in $\mathrm{H}$. If we trace out the subsystem $I I$, we associate the density matrix $\rho_{I}(U)$ to this state and thus also the related entanglement entropy $S_{I}(U)$. We can then average through $U$ to get the average en- tanglement entropy ${ }^{1}$ of the subsystem $I$,

$$
S_{I}=\left\langle S_{I}(U)\right\rangle_{\text {average through } U} .
$$

Page conjectured, as was later proven by Sen [8], that

$$
\begin{aligned}
S_{I}= & \sum_{k=n+1}^{N} \frac{1}{k}-\frac{m-1}{2 n}, \quad \text { for } m \leq n,(4) \\
& \sum_{k=m+1}^{N} \frac{1}{k}-\frac{n-1}{2 m}, \quad \text { for } m \geq n .(5)
\end{aligned}
$$

Notice the symmetry under $n \leftrightarrow m$ of the first and second expression. This is a manifestation of the general result, $S_{I}=S_{I I}$, valid for an overall pure system. Here this means that, when the observable d.o.f., $m$, become bigger than the unobservable d.o.f., $n$, then one can reverse the point of view, and use $S_{I I}$ instead. Some of the mathematical details of this construction can be seen in [7, where this was applied to black hole (BH) evaporation (see also the review [9]). In that application, it is assumed that $\mathrm{BH}$ evaporation takes an initial pure state into a final pure state, while middle states are mixed. The total Hilbert space is factorized into a product, where the subsystem $I$ corresponds to the unobserved states under the horizon, and the subsystem $I I$ corresponds to the observed Hawking radiation. When the $\mathrm{BH}$ is formed, there is no radiation outside and, hence, $m=1$ and $n=N$, thus, $S_{I}$ is trivially zero (pure state). As the $\mathrm{BH}$ evaporates, $N=n m$ is constant, but $m$ increases while $n$ decreases, and, according to (4), $S_{I}$ increases. Approximately at half time of the evaporation the two subsystems have the same quantum dimensions, $n=m$, the information stored below the horizon starts to leak from the $\mathrm{BH}$ decreasing $S_{I}$, according to (5). When the BH fully evaporates, $n=1$ and $m=N$ and $S_{I}$ returns to zero (pure state).

In Fig. 1 we plot two such processes for two different $N$ s. The figure shows that, for a fixed

\footnotetext{
${ }^{1}$ A related important quantity is the average information, defined as $i \equiv \ln m-S_{I}$.
} 


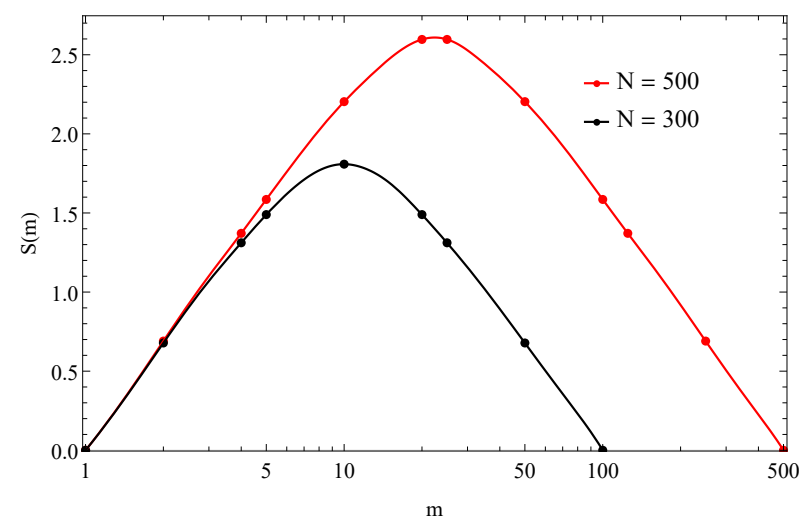

FIG. 1: Example of two Page's curves with total numbers of degrees of freedom 500 and 300. The points are interpolated by spline. Horizontal axis describes entanglement entropy, vertical axis shows the number of degrees of freedom in one part of the bipartite system.

dimension of the observable subsystem, $m, S_{I}$ is bigger for bigger $N$, hence bigger $n$, the unobservable d.o.f. In our application here to nuclear shadowing, we propose that the depth of the region probed by the DIS, that is inversely proportional to $x$, is related to $m$ : the bigger the region probed, the more $m$ are within reach. A full analysis of how this curve might apply to the various regimes of shadowing and antishadowing [3] is beyond the scope of this paper, as we focus on the small $x$ region, and no matter which part of the curve is relevant, what we need to know is that the curve for $N^{\prime}$ is always above the curve for $N$ when $N^{\prime}>N$.

Before moving ahead, let us close this Section by mentioning that different results are obtained for the evolution of a bipartite quantum system such as a $\mathrm{BH}$, when one allows for quasi-particles states (that is non-pure states) as ending states, as shown in [10].

\section{GLUON SHADOWING À LA PAGE}

According to the previous Section, for any given $n$, we have $S_{I}^{\prime}(n)>S_{I}(n)$ when the total dimension of the primed bipartite system, $N^{\prime}=m^{\prime} n$, is bigger than the total dimension of the unprimed bipartite system, $N=m n$. I.e., when, for any given $n$, it is always $m^{\prime}>m$. Let us now recall that for a system of $K$ in- dependent nucleons the global gluon pdf is the product of the free gluon distribution of the single nucleons, i.e.

$$
(x G)_{K}^{\text {free }}=\prod_{i=1}^{K}(x G)_{i}^{\text {free }}
$$

and therefore the corresponding entanglement entropy is given by

$$
S_{K}^{\text {free }}=K S_{N} .
$$

To find the gluon pdf for a nucleus of atomic number $A,(x G)^{A}$, is a nonperturbative and difficult dynamical problem. In this paper a simplified approach is proposed, where a modification of the free nucleon pdf takes into account the nuclear effects. In other words, following Eq.(6), one considers the factorization

$$
(x G)^{A}=\prod_{i=1}^{K}(x G)_{i}^{N / A}
$$

where $(x G)^{N / A}$ is the modified gluon pdf of the single bounded nucleon. Therefore for the nuclear entanglement entropy one obtains

$$
S_{A}=A \ln \left[(x G)^{N / A}\right]
$$

and $S_{A} / A$ is the entanglement entropy per nucleon.

The shadowing effect can now be described in terms of entanglement entropy, since the ratio between the gluon pdfs in nuclei with atomic numbers $A$ and $B$ turns out to be

$$
\exp \left(\frac{S_{A}}{A}-\frac{S_{B}}{B}\right)=\frac{x G^{N / A}}{x G^{N / B}}
$$

This simple model is supported by the following argument on the entanglement of a bipartite systems, when the two parts are modified in different ways by dynamical effects. For a free nucleon, the d.o.f. on the two sides of the separation $I$ and $I I$ are all of the same kind. Whether truly fundamental or not, let us call them "fundamental", as they refer to the simplest nucleon system. Henceforth, each such "fundamental" d.o.f in the observed region $I$ can be entangled at most with one d.o.f. of the 
unobserved region, $I I$, according to the standard monogamy of entanglement. Therefore, depending on how the d.o.f. are shared between $I$ and $I I$, we might have partial or full (maximal) entanglement. Now, suppose that, within the nucleus, the d.o.f. of $I$ and the d.o.f. of $I I$ are affected by the highly nontrivial dynamical effects. Such effects then rearrange the d.o.f. inside the two regions and clearly the pairing between d.o.f. on the two sides is affected too. One might think of such rearrangement as a map from the "fundamental" d.o.f. of the bounded nucleon to the modified d.o.f, of the "quasi-particles". The latter is the picture behind the model presented above.

Let us now discuss the consequences of Eq.(10). If $A>B$, only a reduction of the effective entangled d.o.f. from a larger nucleus to a smaller one can explain the gluon shadowing. Some dynamical mechanisms producing this depletion will be discussed later and in this section we strictly apply Page's formulas to describe gluon shadowing.

Equations (4, 5) can be written in the form

$$
S=\left\{\begin{array}{ll}
\sum_{k=1}^{N} \frac{1}{k}-\frac{m-1}{2 n}-\sum_{k=1}^{n} \frac{1}{k} \quad m \leq n \\
\sum_{k=1}^{N} \frac{1}{k}-\frac{n-1}{2 m}-\sum_{k=1}^{m} \frac{1}{k} \quad m \geq n
\end{array} .\right.
$$

Since for large $N$

$$
\sum_{k=1}^{N} \frac{1}{k} \simeq \ln (N)+\gamma_{M},
$$

where $\gamma_{M}$ is the Eulero-Mascheroni constant, it turns out that

$$
S= \begin{cases}\ln m-\frac{m(m-1)}{2 N} & m \leq \sqrt{N} \\ \ln \frac{N}{m}-\frac{N-m}{2 m^{2}} & m>\sqrt{N}\end{cases}
$$

and

$$
e^{S}= \begin{cases}m \exp \left\{-\frac{m(m-1)}{2 N}\right\} & m \leq \sqrt{N} \\ \frac{N}{m} \exp \left\{-\frac{N-m}{2 m^{2}}\right\} & m>\sqrt{N}\end{cases}
$$

The result in eq. (2) has been obtained by considering a bipartite system (observed and unobserved part of the free nucleon wave function). From this point of view Page's general formulas can be applied, giving a relation among the d.o.f. $m$ ( or $n$ ), $N$ and the free nucleon gluon pdf. Indeed, for $m<\sqrt{N}$, one has

$$
S_{D}=\ln m_{D}-\frac{m_{D}\left(m_{D}-1\right)}{2 N_{D}}=\ln \left[x G_{D}(x)\right]
$$

where $S_{D}$ and $x G_{D}$ are respectively the entanglement entropy and the gluon pdf per nucleon in deuterium, which we identify with the free nucleon ones, neglecting the small binding effects.

In perturbative QCD $x G(x, Q)$ obeys the BFKL evolution equation [11 and grows at small $x$ as $(1 / x)^{\Delta}$, with $\Delta \simeq 0.15[5]$ at $Q_{0}^{2} \simeq$ $1.7 \mathrm{Gev}^{2}$, and therefore (see eq. (15))

$$
S_{D}=\ln m_{D}-\frac{m_{D}\left(m_{D}-1\right)}{2 N_{D}}=\ln \left[\left(\frac{c}{x}\right)^{\Delta}\right]
$$

where $c=3.34$ is a normalization constant fixed by imposing the phenomenological value of $x G_{D}(x) \simeq 5$ at $x=10^{-4}$ ( see tab.3 of ref. [12] and ref. [13] ).

By previous equation one gets the correpondence between the d.o.f. $m_{D}$ and the $\mathrm{Bj}$ variable $x$ for a free nucleon depicted in fig. 2, which for $N_{D}>>1$ can be approximated by

$m_{D}(x)=\left(\frac{c}{x}\right)^{\Delta}\left[1+\frac{1}{2 N_{D}}\left(\frac{c}{x}\right)^{\Delta}\left(\left(\frac{c}{x}\right)^{\Delta}-1\right)\right]$.

Also for a nuclear target, $A$, DIS probes only part of the bounded nucleon's wave function and therefore the description of shadowing in terms of a different entanglement entropy for a bounded nucleon with respect to the free nucleon case requires that $m_{A}$, the d.o.f. per nucleon in $A$ in eq. 15 compared with $m_{D}$ in eq. (16) has to be such that $S_{A}<S_{D}$ in the small $x$ region.

In fig. 3 the functions $m_{A}(x)$ that fits the phenomenological results on gluon shadowing for $x<0.008$ at $Q_{0}^{2} \simeq 1.7 \mathrm{Gev}^{2}$ reported in ref. [1] for two different nuclei $(\mathrm{C}$ and $\mathrm{Pb})$ are shown for $N_{D}, N_{A}>>1$ and in fig. 4 there is depicted the corresponding ratio $m_{A} / m_{D}$. 


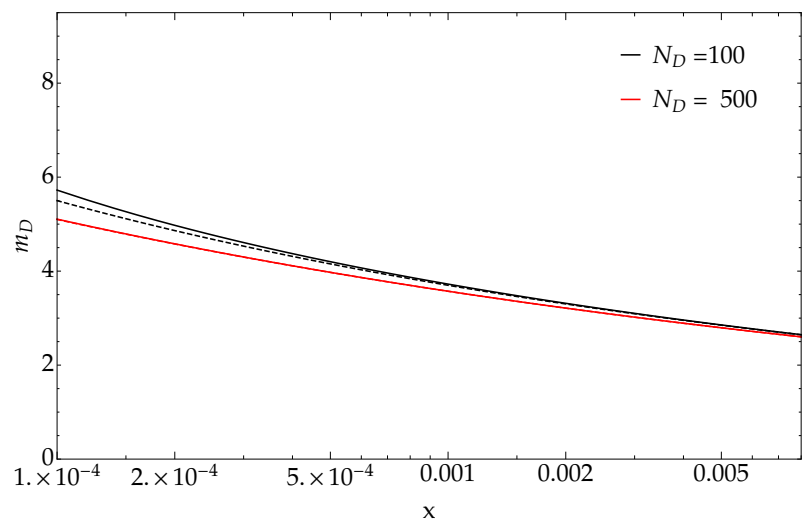

FIG. 2: $m_{D}$ as a function of $x$ from eq. (16) compared with the approximation in eq. (17) for different values of $N_{D}=m_{D} n_{D}$

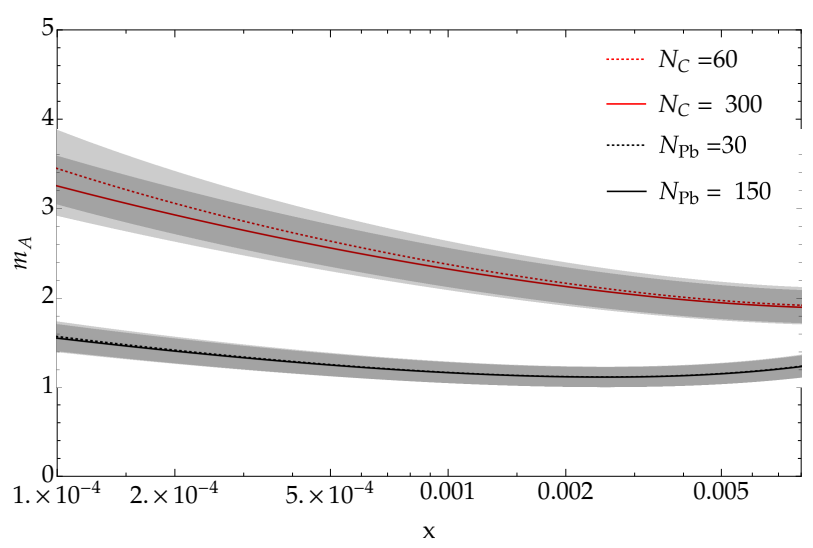

FIG. 3: $m_{A}(x)$ that fits the phenomenological results on gluon shadowing for $x<0.008$ [1 for two different nuclei $(\mathrm{C}$ and $\mathrm{Pb})$ and different values of $N_{C}$ and $N_{C}$. The grey bands are due to the corresponding uncertainties in the gluon distribution function.

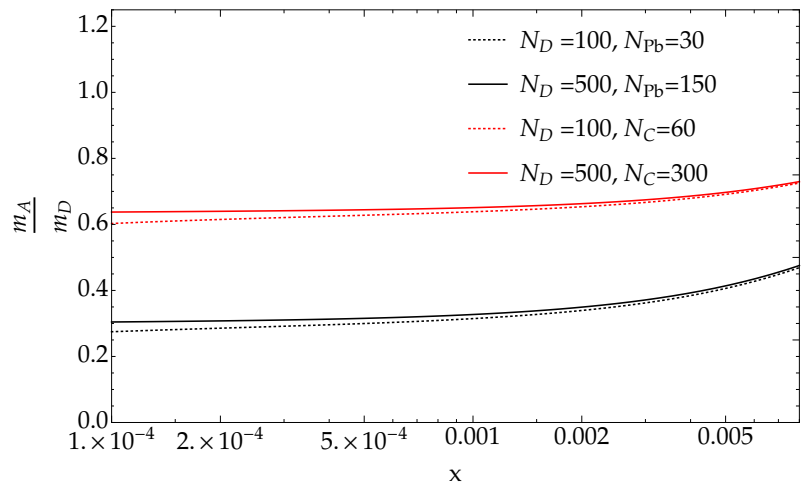

FIG. 4: Ratio $m_{A} / m_{D}$ as a function of $x$ for $\mathrm{C}$ and $\mathrm{Pb}$ and different value od $N_{D}, N_{C}, N_{P b}$

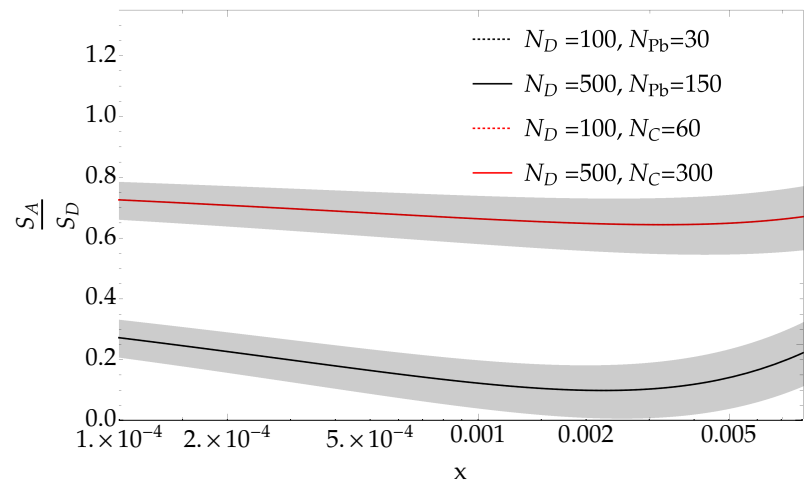

FIG. 5: Ratio $S_{A} / S_{D}$ as a function of $x$ for $\mathrm{C}$ and $\mathrm{Pb}$ and different value od $N_{D}, N_{C}, N_{P b}$. The grey bands are due to the corresponding uncertainties in the gluon distribution function.

The previous analysis clearly shows that:

1. the number of d.o.f. per nucleon probed by DIS in a nucleus is less than the corresponding d.o.f. for a free nucleon, despite the fact that one should expect a larger dimension of the whole Hilbert space for a nucleus.

2. by the results of $m_{A}(x)$ and $m_{D}(x)$ the ratio $S_{A} / S_{D}$ plotted in fig. 5 , turns out to be almost constant in a large range of (small) $x$, with $S_{P B} / S_{D} \simeq 0.3$ and $S_{C} / S_{D} \simeq 0.7$.

\section{DYNAMICAL MECHANICS FOR NUCLEAR ENTANGLEMENT}

The results in Sec. 2 are based on the general Page's framework and points (1-2) call for possible dynamical interpretations.

A possible, simple mechanism is related to the overlap of nucleons in the nucleus [14]: due to the larger overlap in nuclei of atomic number $A$ and $B$, with $A>B$, the observed number of d.o.f. per nucleon decreases which in general implies that unobserved d.o.f. per nucleon increases. By assuming the dominance of two nucleons overlap in nuclei [14] and defining $V_{0}^{A}$ the overlap per nucleon in nucleus $A$, for the ratio $S_{A} / S_{D}$ one expects

$$
S_{A} / S_{D}=1-V_{0}^{A}
$$


The overlapping volume per nucleon has been evaluated in ref. 14 (table I, column b) and for the second member of the previous ratio one gets $\simeq 0.28$ for $P b$ and $\simeq 0.6$ for $C$ which are compatible with the ratios in fig. 5 if one takes into account the large uncertainty band in the gluon pdf.

Another dynamical interpretation of the depletion of d.o.f per bounded nucleon comes from ref. [15] where it has been suggested that the gluon condensate in vacuum, at zero temperature,

$$
<\frac{\alpha_{s}}{\pi} G_{\mu \nu}^{a}(0) G^{\mu \nu a}(0)>
$$

has a large entropy, arising from lumps of condensate with a finite correlation length. On the other hand, the gluon condensate shrinks when the baryon density increases [16, 17] and, despite this result referring to nuclear matter, one can expect this depletion to come out, for instance, by considering light and heavy nuclei. In fact, this depletion of the gluon condensate and consequently of the related entropy has a role in the DIS structure functions. In the small $x$ region the structure function is described by the pomeron exchange [18] and for $x \rightarrow 0$ the structure functions are related to the quarkpomeron effective coupling in the target $(\mathrm{T})$, $\beta_{T}$,

$$
F_{2}(x) \underset{x \rightarrow 0}{\longrightarrow} \beta_{T},
$$

and the ratio of the structure functions per nucleon in the nucleus A with respect to nucleus $\mathrm{B}$ is given by [19]

$$
\frac{F_{2}(x)^{A}}{F_{2}(x)^{B}} \mid \underset{x \rightarrow 0}{\longrightarrow} \frac{\beta_{A}}{\beta_{B}}
$$

On the other hand, in the model proposed by Landshoff and Nachtmann [20] the pomeron exchange is described by nonperturbative gluons and the quark pomeron coupling is related to the gluon condensate according to [21, 22]

$$
\beta_{T} \simeq a^{5}<T\left|\frac{\alpha_{s}}{\pi} G_{\mu \nu}^{a}(0) G^{\mu \nu a}(0)\right| T>.
$$

One can consider the correlation length $a$ as independent of the target, because it is connected to the pomeron coupling to off-shell quarks 21, 22] and therefore one obtains

$\frac{F_{2}(x)^{A}}{F_{2}(x)^{B}} \mid \underset{x \rightarrow 0}{\longrightarrow} \frac{\beta_{A}}{\beta_{B}}=\frac{<A\left|\frac{\alpha_{s}}{\pi} G_{\mu \nu}^{a}(0) G^{\mu \nu a}(0)\right| A>}{<B\left|\frac{\alpha_{s}}{\pi} G_{\mu \nu}^{a}(0) G^{\mu \nu a}(0)\right| B>}$

which suggests the reason for the depletion of entropy in DIS at small $x$ through the nuclear modification of the gluon condensate.

Another interesting aspect is the origin of antishadowing. A dynamical model of antishadowing of the gluon distribution has been proposed in ref. [23], although antishadowing is usually obtained in phenomenological analyses by imposing the energy-momentum sum rule. In the entanglement interpretation of nuclear pdf per nucleon, antishadowing should be related to the conservation of the total entropy, i.e.

$$
\int_{0}^{1} d x S_{A}(x)=\int_{0}^{1} d x S_{D}(x)
$$

which would require a complete description of parton pdf in terms of entanglement entropy in the whole $x$ range. In this respect the calculation of entropy in the valence approximation [24, i.e. for quarks in the color singlet baryon state, gives results consistent with the previous ones, since $S_{B}=\ln 3 \simeq 1.1$ for $x \rightarrow 1$ for a free nucleon (see eq. (3.4) of ref. [24]) and, according to the previous normalization, $S_{D} \simeq 1.6$ for $x=10^{-4}$.

\section{COMMENTS AND CONCLUSIONS}

We put next to each other the evolution of the entanglement entropy of a bipartite system and the phenomenon of the nuclear shadowing. The first phenomenon is behind the famous Page curve of black-hole evaporation, in the unitary picture [6, 7], while the latter nuclear phenomenon refers to the depletion of the parton distribution functions per nucleon within a nucleus, with respect to the free nucleon. Relying on [4], where entanglement entropy and parton distribution functions are found to be related, we wanted to move here the first steps towards understanding gluon shadowing in that approach. We leave it for future work to use more refined versions of Page curves [10. 
At first sight, the Page curves point to a behavior that clashes with shadowing as the entanglement entropy increases for larger unobserved degrees of freedom (i.e., larger nuclei). On the other hand, we have focused on the small $x$ region and we have presented indications that the shadowing might indeed be explained as due to a depletion induced by the overlapping volume per nucleon in a nucleus: the larger the overlap (hence, the larger the nucleus), the less degrees of freedom entangle.

Of course the proposed approach is just indicative and no detailed description of gluon shadowing could be obtained at this stage. Nonetheless, we are able to successfully compare our formulae with the EPPS16 fit of gluon shadowing for $\mathrm{C}$ and $\mathrm{Pb}$. This shows that the entanglement interpretation of the depletion of gluon distribution in nuclei is a correct first step towards a more detailed dynamical description.

\section{Acknowledgements}

A.I. is partially supported by UNCE/SCI/013.
[1] K.J. Eskola , P. Paakkinen , H. Paukkunen, C.A. Salgado, Eur. Phys. J. C 77 (2017) 163.

[2] K. Kovaric et al., Phys.Rev. D 93 (2016) 085037.

[3] N. Armesto, J. Phys. G: Nucl. Part. Phys. 32 (2006) R367.

[4] D.E. Kharzeev, E.M. Levin, Phys.Rev. D 95 (2017) 114008.

[5] M.Hentschinski, A.S.Vera and C.Salas, Phys.Rev. D87 (2013) no.7, 076005

[6] D. N. Page, Phys. Rev. Lett. 71 (1993) 1291.

[7] D. N. Page, Phys. Rev. Lett. 71 (1993) 3743.

[8] S. Sen, Phys. Rev. Lett. 77 (1996) 1.

[9] D. Harlow, Rev. Mod. Phys. 88 (2016) 015002.

[10] G. Acquaviva, A. Iorio, M. Scholtz, Ann. Phys. 387 (2017) 317, PoS (CORFU2017) 206 (2018) 1; A. Iorio, J. Phys.: Conf. Ser. 1275 (2019) 012013.

[11] L. N. Lipatov, Sov. J. Nucl. Phys. 23 (1976) 338, E. A. Kuraev, L. N. Li- patov, V. S. Fadin, Phys. Lett. B 60 (1975) 50, Sov. Phys. JETP 44 (1976) 443, Sov. Phys. JETP 45 (1977) 199. I. I. Balitsky, L. N. Lipatov, Sov. J. Nucl. Phys. 28 (1978) 822

[12] M.R. Pelicer, E.G. de Oliveira, A.D. Martin and M.G. Ryskin, Eur.Phys.J. C79 (2019) no.1, 9, arXiv:1810.05573.

[13] R.Wallny, "A Measurement of the Gluon Dis- tribution in the Proton and of the Strong Coupling Constant from Inclusive Deep-Inelastic Scattering", PhD thesis, Zurich UNiversity, 2001.

[14] F. E. Close, R. L. Jaffe, R. G. Roberts and G. G. Ross, Phys.Rev. D31 (1985) 1004.

[15] J.Cornwall, Modern Physics Letters A 27, 1230011 (2012).

[16] E. G. Drukarev, M. G. Ryskin, V. A. Sadovnikova, Prog. Part. Nucl. Phys. 47 (2001) 73

[17] M. Baldo, P. Castorina and D. Zappala', Nucl. Phys. A743 (2004) 3.

[18] P. Landshoff and J. C. Polkinghorne, Phys. Rep. 5 (1972) 1.

[19] P. Castorina and A. Donnachie, Phys. Lett. B 215 (1988) 589.

[20] P. V. Landshoff and O. Nachtmann, Z. Phys. C 35 (1987) 405.

[21] A. Donnachie, P. V. Landshoff, Nucl. Phys. B311 (1988) 509.

[22] J. R. Cudell, A. Donnachie, P. V. Landshoff, Nucl. Phys. B322 (1989) 55.

[23] L. Frankfurt, V. Guzey and M. Strikman, Phys. Rev. C 95, 055208 (2017).

[24] David E. Miller, Eur.Phys.J. C34 (2004) 435437. 